\title{
Simulations of fully deformed oscillating flux tubes *
}

\author{
K. Karampelas and T. Van Doorsselaere
}

\begin{abstract}
Centre for Mathematical Plasma Astrophysics, Department of Mathematics, KU Leuven, Celestijnenlaan 200B bus 2400, 3001 Leuven, Belgium

e-mail: kostas. karampelas@kuleuven. be
\end{abstract}

Received 25 July 2017 / Accepted 15 December 2017

\begin{abstract}
Context. In recent years, a number of numerical studies have been focusing on the significance of the Kelvin-Helmholtz instability in the dynamics of oscillating coronal loops. This process enhances the transfer of energy into smaller scales, and has been connected with heating of coronal loops, when dissipation mechanisms, such as resistivity, are considered. However, the turbulent layer is expected near the outer regions of the loops. Therefore, the effects of wave heating are expected to be confined to the loop's external layers, leaving their denser inner parts without a heating mechanism.

Aims. In the current work we aim to study the spatial evolution of wave heating effects from a footpoint driven standing kink wave in a coronal loop.

Methods. Using the MPI-AMRVAC code, we performed ideal, three dimensional magnetohydrodynamic simulations of footpoint driven transverse oscillations of a cold, straight coronal flux tube, embedded in a hotter environment. We have also constructed forward models for our simulation using the FoMo code.

Results. The developed transverse wave induced Kelvin-Helmholtz (TWIKH) rolls expand throughout the tube cross-section, and cover it entirely. This turbulence significantly alters the initial density profile, leading to a fully deformed cross section. As a consequence, the resistive and viscous heating rate both increase over the entire loop cross section. The resistive heating rate takes its maximum values near the footpoints, while the viscous heating rate at the apex.

Conclusions. We conclude that even a monoperiodic driver can spread wave heating over the whole loop cross section, potentially providing a heating source in the inner loop region. Despite the loop's fully deformed structure, forward modelling still shows the structure appearing as a loop.
\end{abstract}

Key words. magnetohydrodynamics (MHD) - Sun: corona - Sun: oscillations

\section{Introduction}

Heating of coronal loops by transverse magnetohydrodynamic (MHD) waves has been an extensively studied topic ever since the proof of their ubiquity in the solar atmosphere (Aschwanden et al. 1999; Tomczyk et al. 2007). The main theory of wave damping is resonant absorption for the case of standing modes (Ionson 1978; Goossens et al. 1992, 2011; Arregui et al. 2005; Terradas et al. 2010) and its analogous mechanism of mode coupling (Pascoe et al. 2010; De Moortel et al. 2016) for propagating waves. In both mechanisms the energy of the large-scale oscillation is transferred, through resonance, to local azimuthal Alfvén modes. In the case in which multiple frequencies are excited, smaller scales are created through phase mixing (Heyvaerts \& Priest 1983; Soler \& Terradas 2015). By including dissipation mechanisms, such as resistivity or viscosity, resonant absorption and mode coupling can lead to heating (Ofman et al. 1998; Pagano \& De Moortel 2017).

However, the effects of wave heating by global oscillations were believed to be confined in the resonant layer. As shown in Cargill et al. (2016), this localised heating is not capable of sustaining a fixed density gradient between the loop and the environment. Radiative cooling will inevitably lead to draining of

\footnotetext{
${ }^{\star}$ A movie attached to Fig. 1 is available at http://www . aanda.org
}

the loop's denser inner parts, unless additional heating mechanisms are considered. A possible solution could be the use of a broad-band driver for transverse waves. In such a case (Ofman et al. 1998), we would see the development of multiple narrow resonance layers. These layers can move across the loop crosssection as the density profile changes, but heating would still be concentrated in near-discrete locations.

The previous issue could also be potentially addressed by the Kelvin-Helmholtz instability (KHI) for standing modes in closed coronal structures (Heyvaerts \& Priest 1983; Zaqarashvili et al. 2015). Its existence is predicted by three dimensional simulations in straight flux tubes for driver generated azimuthal Alfvén waves (Ofman et al. 1994; Poedts et al. 1997), impulsively excited standing kink modes (Terradas et al. 2008; Antolin et al. 2014; Magyar et al. 2015; Magyar \& Van Doorsselaere 2016; Howson et al. 2017), and footpoint driven standing kink modes (Karampelas et al. 2017). The KHI creates a turbulent layer at the loop edges, where resonant absorption and phase mixing can effectively transfer energy to smaller scales. However, even if enough energy is provided to the system, its heating would still be mainly localised in the edge of the loop.

Recently, decayless low-amplitude kink oscillations have been discovered in coronal loops (Nisticò et al. 2013; Anfinogentov et al. 2015). The KHI could play an important role in this physical phenomenon, since the observations suggest that the decayless waves are also standing waves with an 
average amplitude of $\sim 0.2 \mathrm{Mm}$, but lower than $1 \mathrm{Mm}$. Antolin et al. (2016) have proposed line-of-sight (LOS) effects due to the $\mathrm{KHI}$ and limits in the spatial resolution of our observations, as the cause of this observed decayless motion. Another proposal is the development of standing waves through a driving mechanism near the loop footpoints (Nakariakov et al. 2016), like those simulated in Karampelas et al. (2017).

In the current study, we have expanded on our previous work, aiming to model the low-amplitude, decayless kink waves in active region coronal loops, driven by footpoint motions. In our previous study (Karampelas et al. 2017), we had concentrated on the longitudinal dependence of the heating rate by the transverse wave induced Kelvin-Helmholtz (TWIKH) rolls. Here, however, we have concentrated on an interesting result about the spatial evolution of a loop cross section for standing kink waves generated by footpoint drivers. In particular, we are going to study the effects of the TWIKH rolls on the cross-sectional density profile and the location of wave heating in the cross section of the loop.

\section{Numerical model}

We simulate footpoint-driven transverse waves in a 3D straight, density-enhanced magnetic flux tube, in a low- $\beta$ coronal environment. Our setup follows the work of Karampelas et al. (2017), with a loop length $L=200 \mathrm{Mm}$ and radius $R=1 \mathrm{Mm}$. The loop density is equal to $\rho_{\mathrm{i}}=2.509 \times 10^{-12} \mathrm{~kg} \mathrm{~m}^{-3}$, and the loop temperature is $T_{\mathrm{i}}=9 \times 10^{5} \mathrm{~K}$. The index $\mathrm{i}$ (e) denotes internal (external) values. The magnetic field is $B_{z}=22.8 \mathrm{G}$ and the plasma $\beta=0.018$. The density profile consists of the continuous function:

$\rho(x, y)=\rho_{\mathrm{e}}+0.5\left(\rho_{\mathrm{i}}-\rho_{\mathrm{e}}\right)\left\{1-\tanh \left(\left(\frac{\sqrt{x^{2}+y^{2}}}{R}-1\right) b\right)\right\}$,

where $x$ and $y$ denote the co-ordinates in the plane perpendicular to the loop axis, $z$ along its axis. For $b=20$ the inhomogeneous layer has a width $\ell \approx 0.3 R$. The density ratio of $\rho_{\mathrm{e}} / \rho_{\mathrm{i}}=1 / 3$, inspired by observational data in Aschwanden et al. (2003), leads to swift transfer of energy from transverse to azimuthal motions. In Karampelas et al. (2017) it was found that the dynamics of the oscillations are not sensitive to the value of the temperature ratio. In the current setup we have effectively modelled a coronal loop during its cooling phase, by choosing a gradient of $T_{\mathrm{i}} / T_{\mathrm{e}}=$ $1 / 3$. The external and internal Alfvén speeds are equal to $v_{\mathrm{Ae}}=$ $2224 \mathrm{~km} \mathrm{~s}^{-1}$ and $v_{\mathrm{Ai}}=1284 \mathrm{~km} \mathrm{~s}^{-1}$.

As in the previous study, we have used the MPI-AMRVAC code (Porth et al. 2014), with an effective resolution of $512 \times 256 \times 64$. Since our domain dimensions are $(x, y, z)=$ $(16,8,100) \mathrm{Mm}$, the cell dimensions are $31.25 \times 31.25 \times$ $1562.5 \mathrm{~km}$. The numerical resistivity is estimated to correspond to a Lundquist number of $S \geq 2.1 \times 10^{4}$.

As before, the tube is driven from the footpoint $(z=0 \mathrm{Mm})$ with the kink period of $P \simeq 2 L / c_{\mathrm{k}} \simeq 254 \mathrm{~s}$ (Edwin \& Roberts 1983). The driver velocity, at the bottom boundary, is uniform inside the loop and time varying as follows:

$\left\{v_{x}, v_{y}\right\}=\{v(t), 0\}=\left\{v_{0} \cos (2 \pi t / P), 0\right\}$,

where $v_{0} \mathrm{~km} \mathrm{~s}^{-1}$ is the peak velocity amplitude. We considered two different cases, one for a driver with a peak velocity of $v_{0}=$ $2 \mathrm{~km} \mathrm{~s}^{-1}$, and one with $v_{0}=0.8 \mathrm{~km} \mathrm{~s}^{-1}$. Outside the loop, the velocity follows the relation:

$\left\{v_{x}, v_{y}\right\}=v(t) R^{2}\left\{\frac{x^{2}-y^{2}}{\left(x^{2}+y^{2}\right)^{2}}, \frac{2 x y}{\left(x^{2}+y^{2}\right)^{2}}\right\}$,
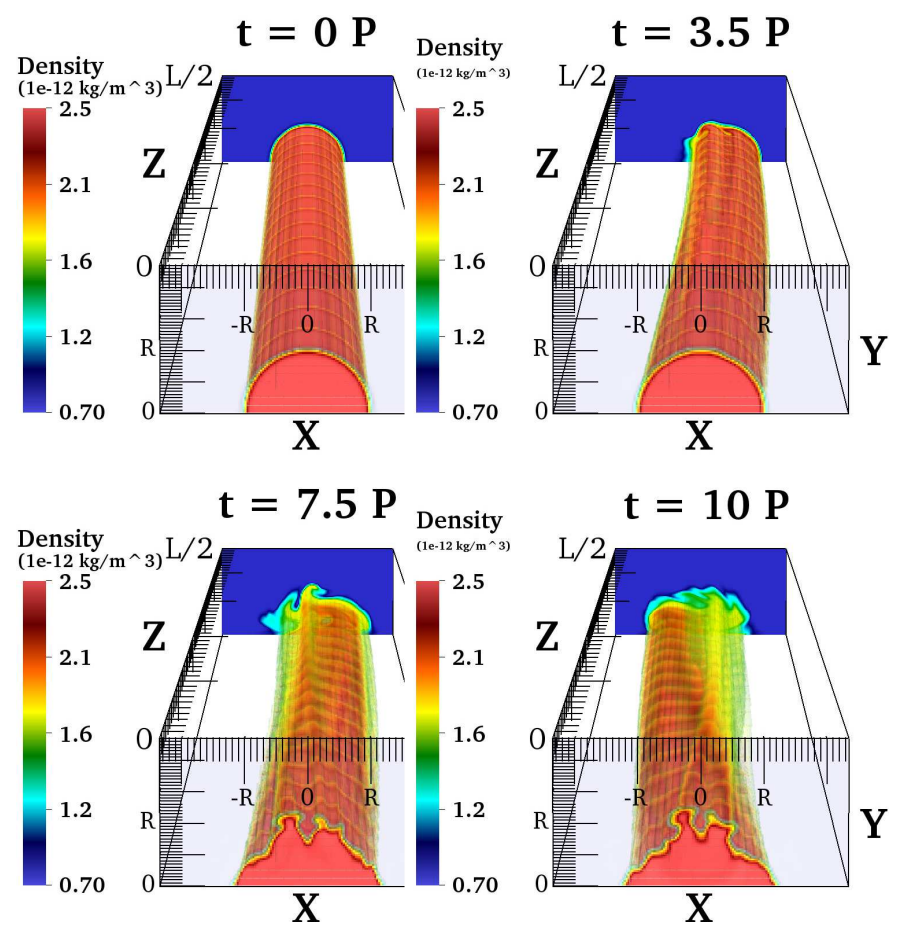

Fig. 1. Density structure of the flux tube, for a driver with $v_{0}=2 \mathrm{~km} \mathrm{~s}^{-1}$. Snapshots are taken for times $t=0,3.5 P, 7.5 P$, and $10 P$, where $P \simeq$ $254 \mathrm{~s}$ is the driver period. The loop length is $L=200 \mathrm{Mm}$ and the loop radius is $R=1 \mathrm{Mm}$. An animation of this figure, showing the oscillation for our model, is available online.

with a smooth transition region between the two areas, matching the outer layer of the cylindrical tube. Following Karampelas et al. (2017), we also set the velocity component parallel to the $z$ axis $\left(v_{z}\right)$ antisymmetric at the bottom boundary, to prevent mass flow through it. The driver sets the values for $v_{x}$ and $v_{y}$ at the bottom boundary, while all the other quantities obey a Neumann-type, zero-gradient condition there. Using the given driver frequency ensures that the superposing propagating waves (originating from each footpoint) form the fundamental standing kink mode for our tube. Taking advantage of the symmetric nature of the kink mode, we kept $v_{z}, B_{x}$, and $B_{y}$ antisymmetric in the $x-y$ plane at the top boundary (apex, $z=100 \mathrm{Mm}$ ), while all the other quantities are symmetric. Furthermore, through the symmetric nature of our driver we set $v_{y}$ and $B_{y}$ antisymmetric in the $x-z$ plane, while the other quantities are symmetric. Therefore, we only simulated one fourth of our tube, as shown in Fig. 1. In the rest of the boundaries, a zero-gradient condition was used for all the quantities.

\section{Results}

For the rest of our analysis we focus on the sub-region of our computational domain, defined by $0 \leq z \leq 100 \mathrm{Mm},|x| \leq$ $2.33 \mathrm{Mm}$ and $y \leq 2.33 \mathrm{Mm}$, where the resolution is the highest. We ran a simulation for a total time of ten driving periods $(10 P \sim 2540 \mathrm{~s})$.

The loop apex, which is the location of the antinode of the $x$ velocity, is Kelvin-Helmholtz unstable, as expected from theory (Heyvaerts \& Priest 1983; Zaqarashvili et al. 2015). As we see in Fig. 2, the KHI manifests at the apex. In addition to that, we have the formation of spatially extended eddies, the TWIKH rolls. A similar feature can be seen in Fig. 3, where we show the density cross-section of our tube at the apex for the driver with peak 

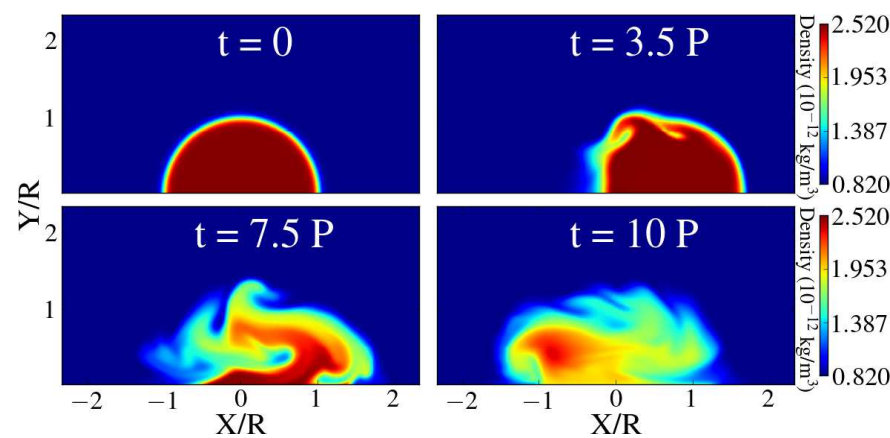

Fig. 2. Snapshots of density $\left(10^{-12} \mathrm{~kg} \mathrm{~m}^{-3}\right)$ of the flux tube cross-section at the apex $(z=100 \mathrm{Mm})$, for a driver with $v_{0}=2 \mathrm{~km} \mathrm{~s}^{-1} . P \simeq 254 \mathrm{~s}$ is the driver period.
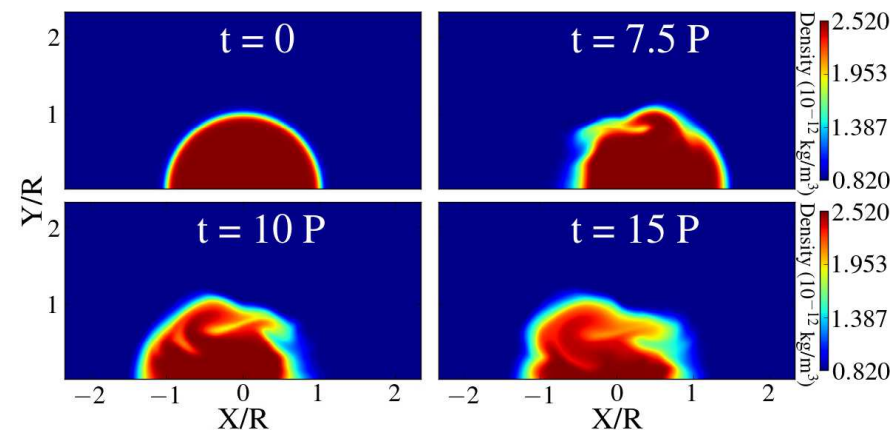

Fig. 3. Same as Fig. 2, but for a driver with $v_{0}=0.8 \mathrm{~km} \mathrm{~s}^{-1}$.

velocity $v_{0}=0.8 \mathrm{~km} \mathrm{~s}^{-1}$. The TWIKH rolls are now less prominent, due to the smaller driver velocity amplitude, which leads to slower increase of the shear velocities at the apex. However, letting the driver act for more periods also leads to a deformed cross section at the apex.

By performing forward modelling with the FoMo code (Van Doorsselaere et al. 2016), we observe the manifestation of strand-like structures due to the out-of-phase movements of the TWIKH rolls, which we also see in Fig. 1. These strands, resembling those in Antolin et al. (2016) for the impulsively excited standing kink waves, are depicted in Fig. 4. Here, we present snapshots of the emission intensity for the Fe XII 193.509 $\AA$ line, at times $t=0,3.5 P, 7.5 P$, and $10 P$. In our setup, the chosen line is better suited to detect the hotter plasma at the loop edge (Antolin et al. 2017). The colour-scale is limited between the minimum and the maximum intensity values of the integrated intensity of the simulations. We consider a LOS plane perpendicular to the loop axis and we set the LOS angle perpendicular to the oscillation direction equal to $0^{\circ}$. By choosing the given LOS angle and studying only the emission intensity, we avoid any missed emission from performing forward modelling in only half the loop cross-section.

In Fig. 5, we plot the average density, temperature, resistive heating rate $\left(H_{\mathrm{res}}\right)$ and the heating by shear viscosity (traced here by square $z$-vorticity, $\omega_{z}^{2}$ ), as functions of distance from the centre of mass, both at the apex and near the footpoint. Initially, the values of $H_{\text {res }}$ and $\omega_{z}^{2}$ are zero, and remain small for the first few periods. At the apex, both the density and temperature spread across the cross section, as a result of the extended TWIKH rolls, effectively widening the loop boundary layer. Near the footpoint, suppression of the KHI results in less mixing, which is evident from the corresponding density and temperature profiles. The resistive heating rate peaks near the footpoint, in agreement with
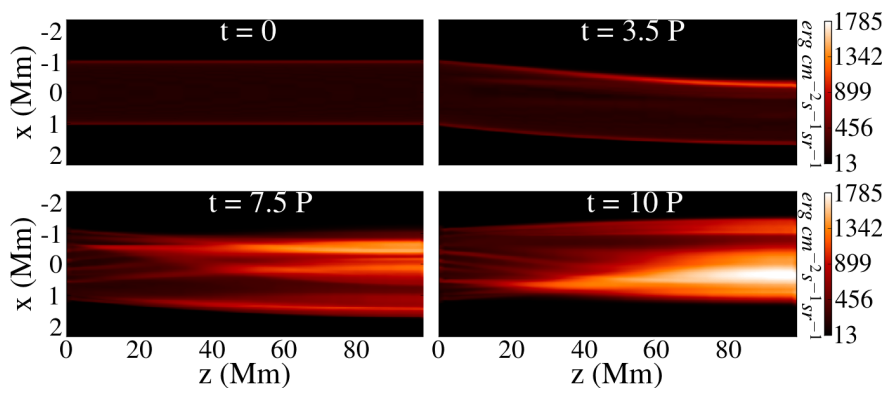

Fig. 4. Forward modelling images of the integrated emission intensity (in erg $\mathrm{cm}^{-2} \mathrm{~s}^{-1} \mathrm{sr}^{-1}$ ) of the tube for the 193.509 $\AA$ line. The observer is at a $0^{\circ}$ LOS angle, perpendicular to the oscillatory motion. Half the loop length is modelled $(z=0-100 \mathrm{Mm})$. The driver peak velocity is $v_{0}=2 \mathrm{~km} \mathrm{~s}^{-1}$, and $P \simeq 254 \mathrm{~s}$ is the driver period.

Karampelas et al. (2017). On the other hand, the viscous heating rate peaks near the loop. Radially, both of them are initially confined to the resonant layer, but later spread out over the entire loop cross section, as we can see in Fig. 6 for the $\omega_{z}^{2}$ at the apex and the $H_{\text {res }}$ at the footpoint, for snapshots at $t=7.5 P$.

\section{Discussion and conclusions}

Looking at the loop cross section at the apex (Fig. 2), it is interesting to see the gradual deformation of the loop, as the simulation reaches its final stages. In the current model, the site of the highest deformation is located in the area near the loop apex, because the $v_{x}, v_{y}$ velocity antinode and $B_{x}, B_{y}$ magnetic field node appear there. Previous works have shown that TWIKH rolls create a wide turbulent layer both for impulsive (Magyar \& Van Doorsselaere 2016) and driven (Karampelas et al. 2017) standing modes. Here our previous simulations were performed for an extended duration $\left(t_{\max }=10 P\right)$. The extended TWIKH rolls result in a completely deformed loop cross section, where extensive mixing is taking place across the entire loop. Thus the loop cross section at the apex becomes fully deformed. Studying the results in Fig. 3 (and as is intuitively clear), we see that the driver amplitude plays an important role in the development of the KHI and the evolution of the loop cross section. However, we find that the loop cross section also evolves to a fully deformed state, even for the very small driver amplitude $\left(v_{0}=0.8 \mathrm{~km} \mathrm{~s}^{-1}\right)$. Therefore, it is probably safe to assume that coronal loops have a deformed cross section, if they are driven by transverse footpoint motions for a sufficiently long time.

The turbulent nature of the cross section has a profound impact on the radial structure of the loop. As we see from the top panels of Fig. 5, the density and temperature profiles near the apex seem to get smoothed over time, in these angleaveraged radial density and temperature profiles. This effect is most prominent between 50 and $100 \mathrm{Mm}$ (apex). Near the loop footpoint, however, the smearing of density and temperature profiles is less, because the TWIKH rolls are absent there.

The deformation of the loop cross section due to the KHI instability affects the spatial distribution of the resistive and viscous heating rate. Despite the smooth appearance of the density and temperature in Fig. 5, the damping of the kink wave continues to take place. The resonant layer is now turbulently fragmented into many current sheets or shear layers in the velocity, because of the KHI eddies over the whole cross section. The resistive heating peaks near the footpoints, as we see in Fig. 5. 

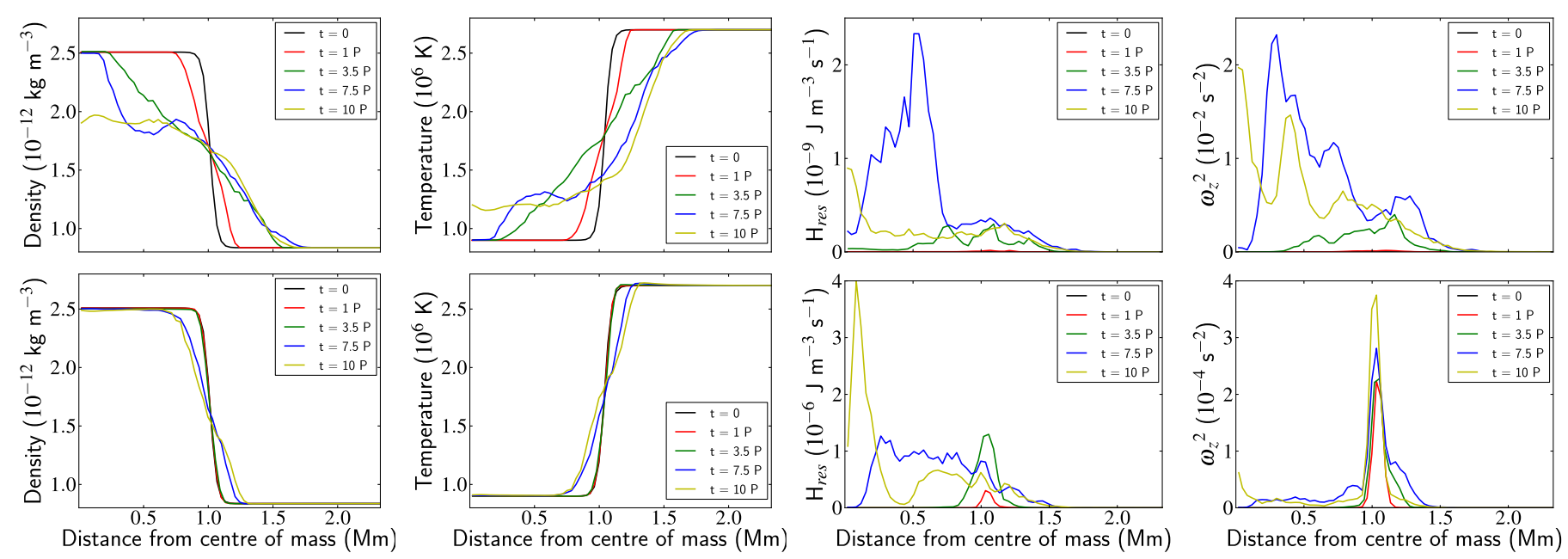

Fig. 5. Profiles of the average density, temperature, resistive heating rate $H_{\text {res }}$ and vorticity $\omega_{z}^{2}$ as a function of the distance from the centre of mass. The driver peak velocity is $v_{0}=2 \mathrm{~km} \mathrm{~s}^{-1}$, and $P \simeq 254 \mathrm{~s}$ is the driver period. The top row shows the profiles at the apex $(z=100 \mathrm{Mm})$. The bottom row shows the profiles near the footpoint $(z=1 \mathrm{Mm})$.

$H_{\text {res }}$, which is dominated by the diffusion of the $J_{z}$ current density (Karampelas et al. 2017), is initially concentrated in the resonant layer. Later, it spreads over the whole tube cross section (Fig. 6), even if the loop is not highly deformed near the footpoint. Instead, this is due to the deformed cross section near the loop top affecting the imprint of the current near the footpoint. Likewise, the viscous heating rate is spreading through the entire cross section because of the turbulent deformation (Fig. 6). However, in contrast to the resistive heating rate, it finds its maximum near the loop apex.

In our current experiments, there is not enough energy input at the footpoint to balance the energy losses which are to be expected from the plasma (by e.g. optically thin radiation or heat conduction). However, in principle the plasma could be heated if a sufficient energy flux is provided by the driven boundary. The key point of this paper is that this heating (be it resistive or viscous) can take place in the entire cross section of the loop, because of its fully deformed nature. While it was earlier refuted by using drivers with a broad-band spectrum, the decade-old argument (Ofman et al. 1998; Cargill et al. 2016) that wave heating can only take place in specific layers in the loop is thus not even true for monoperiodic drivers.

Despite the fully deformed state of the loop cross section, the forward modelling of our simulation (Fig. 4) still maintains a loop-like appearance. The only qualitative changes compared to the forward model of a "laminar" loop (i.e. a straight cylinder) is that (1) strand-like features are formed (as previously pointed out by Antolin et al. 2014), and (2) the overall intensity is increased and spread over a larger layer. The latter is because of the adiabatic expansion during the mixing of the interior and exterior plasma (as previously shown by Antolin et al. 2016; Karampelas et al. 2017). However, the coronal loop structure is clearly distinguishable from its surrounding plasma, and its oscillation remains visible at later times. Thus, the observational identity of a fully deformed loop remains intact, and should be detectable when studying relevant phenomena, such as the decayless loop oscillations from Anfinogentov et al. (2015).

The fact that the heating by the transverse waves occurs in the entire, deformed loop cross section, provides impetus to more
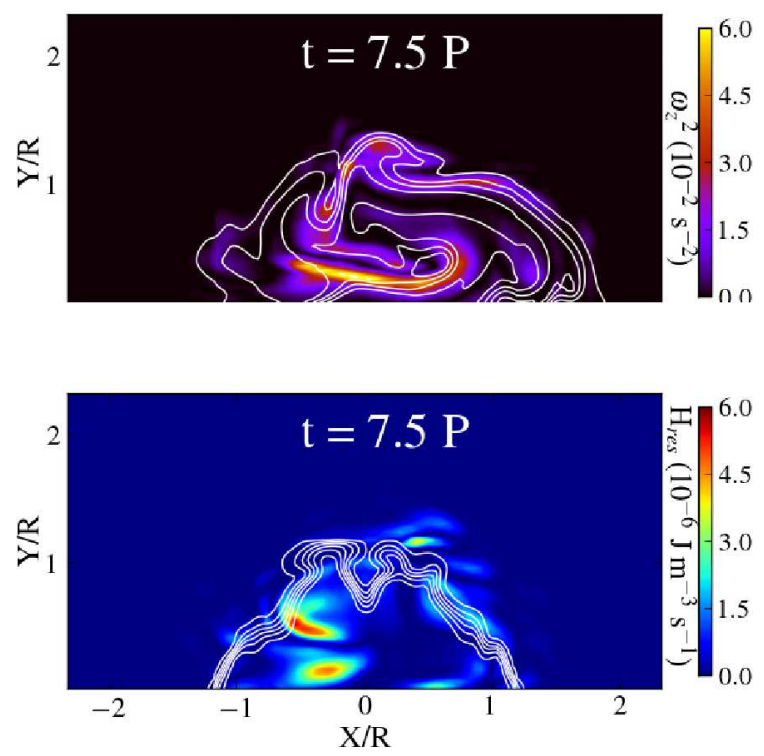

Fig. 6. Snapshots of the vorticity $\omega_{z}^{2}$ at the apex (top) and of the resistive heating rate $H_{\text {res }}$ near the footpoint (bottom), for the driver with $v_{0}=$ $2 \mathrm{~km} \mathrm{~s}^{-1} . P \simeq 254 \mathrm{~s}$ is the driver period. The white lines on both panels represent the density contours at the corresponding heights, showing the circumference of the dense loop.

detailed wave heating models, going beyond the qualitative arguments presented in this paper. The introduction of a realistic atmosphere and thermal conduction in future setups, the inclusion of physical dissipation terms, such as anomalous resistivity, and the use of stronger drivers could provide a viable loop heated by transverse waves in its entirely deformed cross section, further addressing the issues brought up by Cargill et al. (2016).

Acknowledgements. We would like to thank the referee, whose review helped us improve the manuscript. We also thank the editor, for his comments. K.K. was funded by GOA-2015-014 (KU Leuven). T.V.D. was supported by the IAP P7/08 CHARM (Belspo) and the GOA-2015-014 (KU Leuven). This project has received funding from the European Research Council (ERC) under the European Union's Horizon 2020 research and innovation programme (grant agreement No. 724326). The results were inspired by discussions at the ISSI-Bern and at ISSI-Beijing meetings. 
K. Karampelas and T. Van Doorsselaere: Simulations of fully deformed oscillating flux tubes

\section{References}

Anfinogentov, S. A., Nakariakov, V. M., \& Nisticò, G. 2015, A\&A, 583, A136 Antolin, P., Yokoyama, T., \& Van Doorsselaere, T. 2014, ApJ, 787, L22

Antolin, P., Moortel, I. D., Doorsselaere, T. V., \& Yokoyama, T. 2016, ApJ, 830, L22

Antolin, P., De Moortel, I., Van Doorsselaere, T., \& Yokoyama, T. 2017, ApJ 836, 219

Arregui, I., Van Doorsselaere, T., Andries, J., Goossens, M., \& Kimpe, D. 2005, A\&A, 441, 361

Aschwanden, M. J., Fletcher, L., Schrijver, C. J., \& Alexander, D. 1999, ApJ, 520,880

Aschwanden, M. J., Nightingale, R. W., Andries, J., Goossens, M., \& Van Doorsselaere, T. 2003, ApJ, 598, 1375

Cargill, P. J., De Moortel, I., \& Kiddie, G. 2016, ApJ, 823, 31

De Moortel, I., Pascoe, D. J., Wright, A. N., \& Hood, A. W. 2016, Plasma Phys. Control. Fus., 58, 014001

Edwin, P. M., \& Roberts, B. 1983, Sol. Phys., 88, 179

Goossens, M., Hollweg, J. V., \& Sakurai, T. 1992, Sol. Phys., 138, 233

Goossens, M., Erdélyi, R., \& Ruderman, M. S. 2011, Space Sci. Rev., 158, 289

Heyvaerts, J., \& Priest, E. R. 1983, A\&A, 117, 220

Howson, T. A., De Moortel, I., \& Antolin, P. 2017, A\&A, 602, A74
Ionson, J. A. 1978, ApJ, 226, 650

Karampelas, K., Van Doorsselaere, T., \& Antolin, P. 2017, A\&A, 604, A130

Magyar, N., \& Van Doorsselaere, T. 2016, A\&A, 595, A81

Magyar, N., Van Doorsselaere, T., \& Marcu, A. 2015, A\&A, 582, A117

Nakariakov, V. M., Anfinogentov, S. A., Nisticò, G., \& Lee, D.-H. 2016, A\&A, 591, L5

Nisticò, G., Nakariakov, V. M., \& Verwichte, E. 2013, A\&A, 552, A57

Ofman, L., Davila, J. M., \& Steinolfson, R. S. 1994, Geophys. Res. Lett., 21, 2259

Ofman, L., Klimchuk, J. A., \& Davila, J. M. 1998, ApJ, 493, 474

Pagano, P., \& De Moortel, I. 2017, A\&A, 601, A107

Pascoe, D. J., Wright, A. N., \& De Moortel, I. 2010, ApJ, 711, 990

Poedts, S., Toth, G., Belien, A. J. C., \& Goedbloed, J. P. 1997, Sol. Phys., 172, 45

Porth, O., Xia, C., Hendrix, T., Moschou, S. P., \& Keppens, R. 2014, ApJS, 214 4

Soler, R., \& Terradas, J. 2015, ApJ, 803, 43

Terradas, J., Andries, J., Goossens, M., et al. 2008, ApJ, 687, L115

Terradas, J., Goossens, M., \& Verth, G. 2010, A\&A, 524, A23

Tomczyk, S., McIntosh, S. W., Keil, S. L., et al. 2007, Science, 317, 1192

Van Doorsselaere, T., Antolin, P., Yuan, D., Reznikova, V., \& Magyar, N. 2016, Front. Astron. Space Sci., 3, 4

Zaqarashvili, T. V., Zhelyazkov, I., \& Ofman, L. 2015, ApJ, 813, 123 